\title{
Power regulation and electromigration in platinum microwires
}

\author{
Ottó Elíasson, ${ }_{1}^{1}$ Gabriel Vasile, ${ }^{1,2}$ Sigurður Ægir Jónsson, $\left.{ }^{1},{ }^{a}\right)$ G. I. Gudjonsson, ${ }^{1}$ Mustafa Arikan, ${ }^{1}$ and Snorri \\ Ingvarsson ${ }^{1, b)}$ \\ 1) Science Institute, University of Iceland, Dunhagi 3, Reykjavik IS-10\%, Iceland \\ ${ }^{2)}$ National Institute of Research-Development for Cryogenics and Isotopic Technologies, Uzinei 4, \\ Ramnicu Valcea RO-1000, Romania
}

(Dated: 6 November 2018)

\begin{abstract}
We introduce a new experimental setup with a biasing circuit and computer control for electrical power regulation under reversing polarity in $\mathrm{Pt}$ microwires with dimensions of $1 \times 10$ $\mu \mathrm{m}^{2}$. The circuit is computer controlled via a data acquisition board. It amplifies a control signal from the computer and drives current of alternating polarity through the sample in question. Time-to-failure investigations under DC and AC current stress are performed. We confirm that $\mathrm{AC}$ current stress can improve the life time of microwires at least by a factor of $10^{3}$ compared to the corresponding time-to-failure under DC current stress.
\end{abstract}

Keywords: Power regulation, electromigration, microheaters

\section{INTRODUCTION}

Our recent research has involved studying radiative properties of electrically heated microwires in the infrared ${ }^{1-4}$. We often refer to these as microheaters. Biasing with a larger current raises the temperature of the microheater and thus increases the intensity of radiation. It furthermore shifts the radiation spectrum to shorter wavelengths, much like for an ideal blackbody, as described by Planck's law ${ }^{5}$. The details of the spectrum and the wire's resistance at given bias conditions depend on each microheater's physical dimensions. Thus obviously it is important to regulate the temperature in our microheaters.

We have found that to a good approximation the temperature of our microheaters, $T_{\mathrm{h}}$, depends linearly on the power $P_{\mathrm{h}}$ dissipated (Joule heating) in them!

$$
T_{\mathrm{h}}=T_{0}+\frac{d T}{d P} P_{\mathrm{h}}
$$

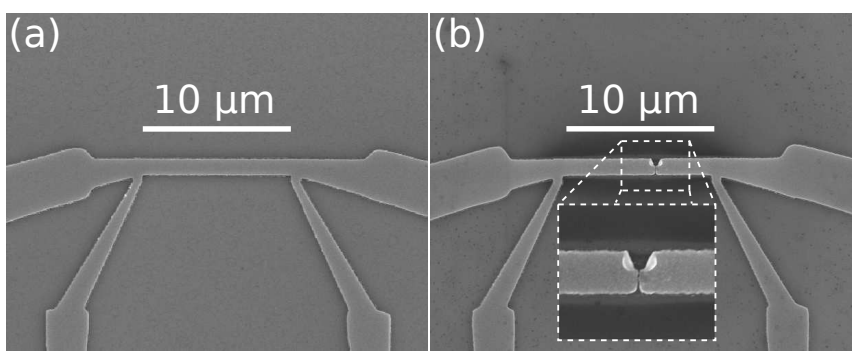

FIG. 1. Scanning electron micrographs of microheaters used in our study (a) before, and (b) after onset of electromigration. A change in the substrate's color surrounding the heater due to heating is apparent. Inset: Englargement of the failure location showing the "mousebite" void, and from it, a narrow slit across to the opposite edge of the wire.

a) Present address: Computational Biology and Biological Physics, Lund University, Sölvegatan 14A, SE-223 62 Lund, Sweden

b) Electronic mail: sthi@hi.is. where $T_{0}$ is room temperature (i.e. $T_{\mathrm{h}}$ at $P_{\mathrm{h}}=0 \mathrm{~mW}$ ). The thermal impedance of the microheater, $d T / d P$, is governed by its surroundings, i.e. the thermal properties of its environment, and remains constant even upon thermal cycling. The resistance of the heaters $R_{\mathrm{h}}$ varies with temperature. Within a narrow enough temperature range around or above room temperature, it may be approximated with the well known relation

$$
R_{\mathrm{h}}=R_{0}(1+\alpha \Delta T)
$$

where $\alpha$ is the temperature coefficient of resistance, $\Delta T=T_{\mathrm{h}}-T_{0}$ and $R_{0}$ is the resistance at room temperature. The electrical resistance is a property of the wire, and depends on its geometry and the material's resistivity. Also we have found that $\alpha$ may in some cases change upon thermal cycling, presumably due to alterations in grain size or other annealing effects. However, the significance of eq. (1) is that it states that if you know the power dissipated in your wire, you can tell its temperature. One can measure $d T / d P$ by current-voltage measurements in a narrow range where $\alpha$ remains unchanged, and subsequently expose the heater to a much broader range of power and temperature confident that $d T / d P$ remains constant even though $\alpha$ may change.

Increased current results in greater Joule dissipation and higher temperature. However, there are limits, and as one reaches a critical current density $\left(J \sim 10^{7} \mathrm{~A} / \mathrm{cm}^{2}\right)$ void formation and electromigration will cause the wire to break, resulting in an open electrial circuit ${ }^{7}$. Electromigration is the process of current-induced self-diffusion in metal lines, and it is the mechanism responsible for longterm wear-out of interconnects in integrated circuits 8 13. Figure 11 displays a typical failure in one of our wires; i.e. there is a "mousebite" void formation and a narrow slit extending from it across the width of the wire ${ }^{14 \mid 15}$. With the carefully tapered contact design, failures typically occur at or near the middle of the length of the wire, with void formation at the edge. The highest temperature occurs in the middle of the wire, but the largest temperature gradient, in-plane perpendicular to the wire, is at the edge midway along the length ${ }^{6}$. This causes large mechanical stress at the edge. For our purposes it is very useful to extend the time-to-failure at a given current bias 
of our microheaters as much as possible. The time-tofailure of the wire $\tau$, can be greatly increased by reversing the current polarity at a frequency $f$ much greater than that corresponding to the time-to-failure of the heater under DC current stress $\tau_{\mathrm{DC}}{ }^{16}$ 18. Our need to maintain constant temperature, thus constant power dissipation, while extending the time-to-failure of our heaters is somewhat unique. It is complicated by the fact that there are slow variations in the wires' resistance values (as discussed below). We were unable to find commercial solutions for this. While communications via the IEEE standard GPIB are sufficiently fast to deal with slow changes in resistance, they are too slow to allow rapid switching of polarity. $\mathrm{D} / \mathrm{A}$ converters provide speed but do not output adequate power in all cases. Thus a special biasing circuit was designed and built, that enables measurement of the power dissipation in the heater itself as well as allowing rapid change of bias polarity. We used computer control to regulate the power and to reverse the current polarity. By reversing the current polarity we also expected to be able to nondestructively reach higher heater temperatures $T_{\mathrm{h}}$ than before.

The outline of this paper will be as follows. We introduce the experimental setup including the circuit and the computer control (the program used for power regulation under AC stressing). We present results of measurements on the time-to-failure of the microheaters comparing $\mathrm{AC}$ and DC current stress and discuss some distinct features observed in the heaters' resistance, $R_{\mathrm{h}}$, as a function of time.

\section{EXPERIMENTAL SETUP}

To bias our microheaters we use a probe station and microprobes to connect to the sample. We employ our new biasing circuit and a programmable National Instruments Data Acquisition board (DAQ-board) of type NIUSB 6229 to regulate the power dissipation in the heater (and thus its temperature) via computer control. The biasing circuit is powered by $\pm V_{\mathrm{CC}}= \pm 15 \mathrm{~V}$ supply voltage.

\section{A. Biasing circuit}

The circuit in fig 2 (initially described in ref. (6) is essentially composed of two followers, each comprised of an operational amplifier (TL072 in our case) and a push-pull transistor amplifier (C557B pnp and C547B npn transistors). The resistors $R_{\mathrm{P}}$ at each tranistor's base are to limit the voltage seen by the base. We use a four point probe connection to our heaters, i.e. two providing current and two to sense voltage. Their contact resistances are represented by $R_{\mathrm{C} 1}, R_{\mathrm{C} 2}, R_{\mathrm{C} 3}$ and $R_{\mathrm{C} 4}$ respectively. The microheater is represented by the resistance $R_{\mathrm{h}}$. Since the inputs of op-amps draw almost no current, the voltage drop in our voltage sensing leads/contacts $\left(R_{\mathrm{C} 2}\right.$ and $\left.R_{\mathrm{C} 3}\right)$ is negligible and the current $I_{\mathrm{h}}$ runs directly through contacts $R_{\mathrm{C} 1}$ and $R_{\mathrm{C} 4}$. The followers ensure that the voltages at points $A$ and $B$ equal the input volt-

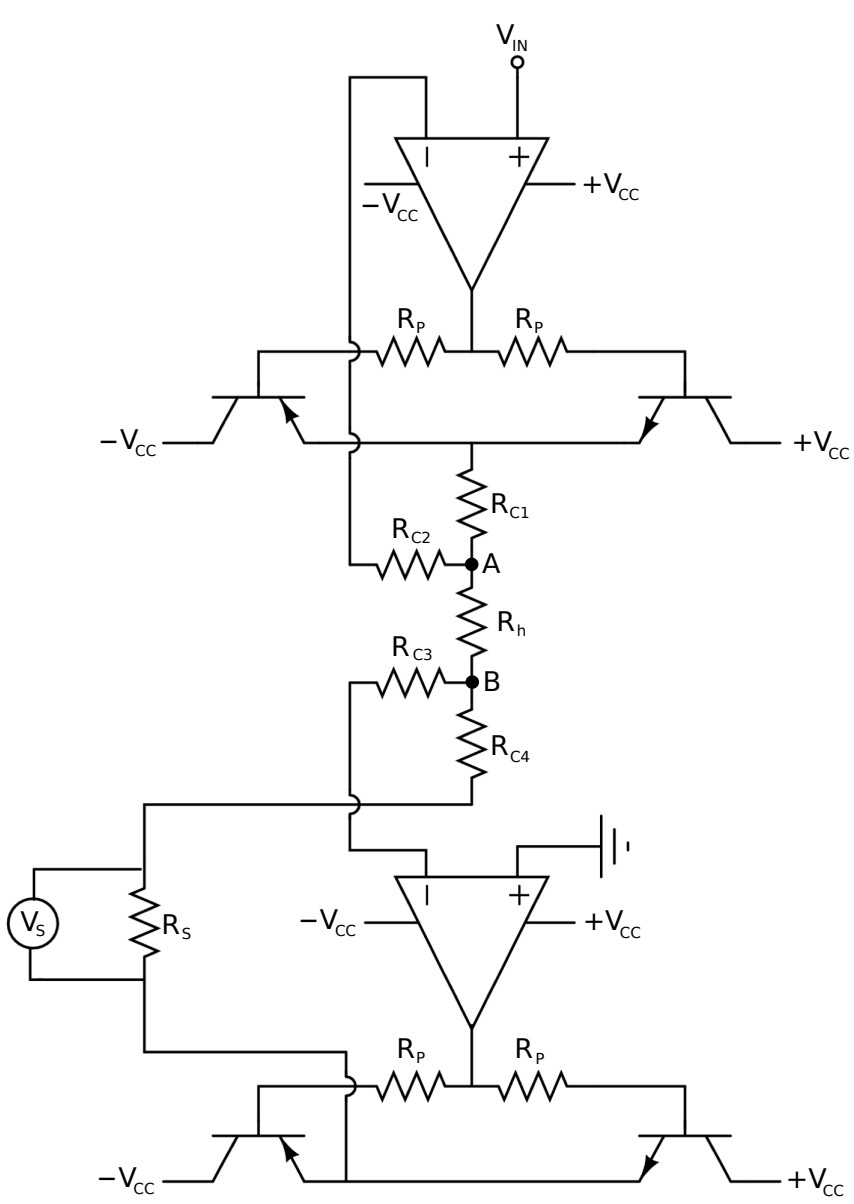

FIG. 2. The circuit that enables monitoring the power dissipation in a microheater and allows rapid switching of bias polarity.

ages $V_{\text {in }}$ and 0 , respectively. Thus we have the relation $I_{\mathrm{h}}=V_{\text {in }} / R_{\mathrm{h}}$. However, as the microheater's resistance increases as it is heated, the current is measured via the voltage drop across a known precision resistor $R_{\mathrm{S}}$. The power dissipation may then be found by

$$
P_{\mathrm{h}}=I_{\mathrm{h}} V_{\mathrm{h}}=\frac{V_{\mathrm{S}}}{R_{\mathrm{S}}} V_{\mathrm{in}}
$$

all known quantities.

It is important to note that if a microheater sample is removed and reconnected there is a high risk of transitents, potentially destructive to the sample. For this reason we turn off the supply voltage and ramp it up in a controlled manner once the microheater is in place.

\section{B. Computer control}

The DAQ-board, which manages the signals to $\left(V_{\text {in }}\right)$ and from $\left(V_{\mathrm{S}}\right)$ the biasing circuit, was programmed in Microsoft's Visual $\mathrm{C}++$ environment for fast response. As the current through the microheater is ramped up we start with a regular proportional "P"-control, where the desired output is manipulated by adjusting the voltage $V_{\text {in }}$ proportional to the "error signal" $P_{\mathrm{h}}-P_{\mathrm{d}}$, where $P_{\mathrm{d}}$ is the desired power value. When $P_{\mathrm{h}}$ approaches the desired value $P_{\mathrm{d}}$ a simpler "fuzzy logic" control takes over 

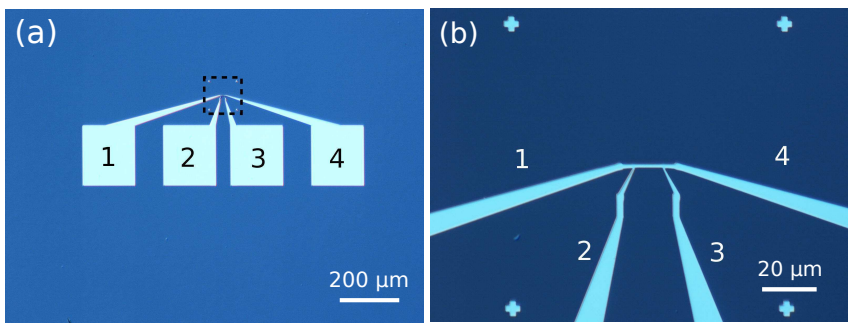

FIG. 3. Optical microscope images of heaters used in this study. The contact pads are labeled with numbers 1-4. Each pad is $180 \times 200 \mu \mathrm{m}^{2}$. The dotted line in fig. (a) demarcates the area pictured in fig. (b).

and maintains $P_{\mathrm{h}}$ at the desired value. An advantage of the fuzzy logic control is that it is not computationally intensive, thus fast. This can easily be replaced by a control of the user's choice, PID or more advanced methods.

In order to combat electromigration we reverse the polarity of the voltage $V_{\text {in }}$ at a frequency $f=20 \mathrm{kHz}$, forming a square wave. This frequency was chosen based on ref. 17 (where the current is regulated, but not the power as in our case), that showed that there was a three order of magnitude lifetime gain by reversing polarity in $\mathrm{Al}$ wires at this rate. However, increasing the frequency by another three orders of magnitude gave a very modest gain in lifetime. Our power regulation, on the other hand, is done at a much lower frequency $f_{r}=200 \mathrm{~Hz}$. This regulation amounts to adjusting the amplitude of the square biasing wave in response to the changes in the microheater's resistance. This much lower frequency of regulation is quite adequate, since the changes in microheater resistance are mostly slow compared with $f_{r}^{-1}$, as can be seen in fig. 4. Although this frequency may be raised somewhat, it is limited by communication and computation time. With this simple method we can easily maintain the power dissipation within $0.1 \%$ of the desired value.

\section{SAMPLE PROPERTIES}

The microheaters used in our study were manufactured on a $\mathrm{Si} / \mathrm{SiO}_{2}$ substrate with e-beam lithography to ensure good shape definition. The heaters, typically about 60 on each substrate chip, are deposited via DC magnetron sputtering. Their structure is simple, a $5 \mathrm{~nm}$ polycrystalline $\mathrm{Cr}$ adhesion layer followed by a $50 \mathrm{~nm}$ polycrystalline Pt layer. The details of our samples are described in ref. 2. In the present study all the microheaters are of the same lateral dimensions $1 \times 10 \mu \mathrm{m}^{2}$. Samples are depicted in fig. 3. In order to make sure that the heaters were as comparable as possible, they were characterized by standard electrical measurements before our experiments. Three quantities describing the properties of our heaters are of special interest. These are the resistance $R_{\mathrm{h}}$ (in particular the cold resistance $R_{c}$ ), the thermal impedance $d T / d P$ and the temperature coefficient of resistance $\alpha$ (prior to thermal cycling, used in the process of determining $d T / d P$ ).

An as-grown (untouched) microheater had a cold re- sistivity of

$$
\rho_{c}=20 \mu \Omega \mathrm{cm},
$$

which is about $90 \%$ greater than many reports of bulk resistivity values of $\mathrm{Pt}$ in the literature $\rho_{\text {bulk }}=10.4 \mu \Omega \mathrm{cm}$. We attribute this deviation primarily to surface scattering, and grain boundary scattering. The TCR value, $\alpha$ was obtained by measuring resistance of heaters sitting on a hot plate whose temperature is increased stepwise resulting in corresponding changes in resistance. We measured over a temperature range from room temperature up to about $110^{\circ} \mathrm{C}$, and found $\alpha$ from fitting eq. (2), to be

$$
\alpha=2.20 \times 10^{-3} \mathrm{~K}^{-1} .
$$

Plotting resistance versus power allows an estimation of $d T / d P$. For our heaters we obtained

$$
\frac{d T}{d P}=4.7 \mathrm{~K} / \mathrm{mW}
$$

After such characterization one heater was subjected to a constant high power for a few minutes, long enough to observe an obvious decrease in the heater's resistance, see below. Following this procedure the heater's $I-V$ profile is obtained again to measure the cold resistance, and its $\alpha$ value is measured again to see if it had changed. No changes were found in either $\alpha$ or $d T / d P$ in our measurements.

\section{TIME-TO-FAILURE MEASUREMENTS}

At low current density and low power the lifetime of our microheaters is, for practical purposes, infinite. However, as bias is increased their lifespan shortens rapidly. We wished to compare time-to-failure of heaters at fixed power under DC and AC stress to see if bias polarity reversal would help. We regulated the power both at a power of $P_{\mathrm{h}}=90 \mathrm{~mW}$ and at $P_{\mathrm{h}}=100 \mathrm{~mW}$, corresponding to about $440^{\circ} \mathrm{C}$ and $490^{\circ} \mathrm{C}$ respectively according to eq. (1) and (6). Typical results are displayed in fig. 4 (a) showing resistance as function of time under DC stress at $P=100 \mathrm{~mW}$, and (b) another identical microheater at the same power but under $\mathrm{AC}$ stress, i.e. a square wave with $f=20 \mathrm{kHz}$. Both show an initial gradual and smooth drop in resistance over a signifant portion of the lifetime, that may be viewed as a "repair process" for the wire. After reaching a minimum the resistance starts to increase, with occasional steps, presumably due to movement of material, i.e. electromigration. Finally, there is a sharp rise in resistance and the wire breaks. Note that the time-to-failure in (b) is more than two orders of magnitude longer than in (a). Measurements of the DC time-to failure of 23 samples resulted in the following mean time-to-failure for $90 \mathrm{~mW}$ and $100 \mathrm{~mW}$ respectively:

$$
\begin{aligned}
\tau_{\mathrm{DC}, 90} & =[4000 \pm 200] \mathrm{s} \\
\tau_{\mathrm{DC}, 100} & =[270 \pm 30] \mathrm{s} .
\end{aligned}
$$



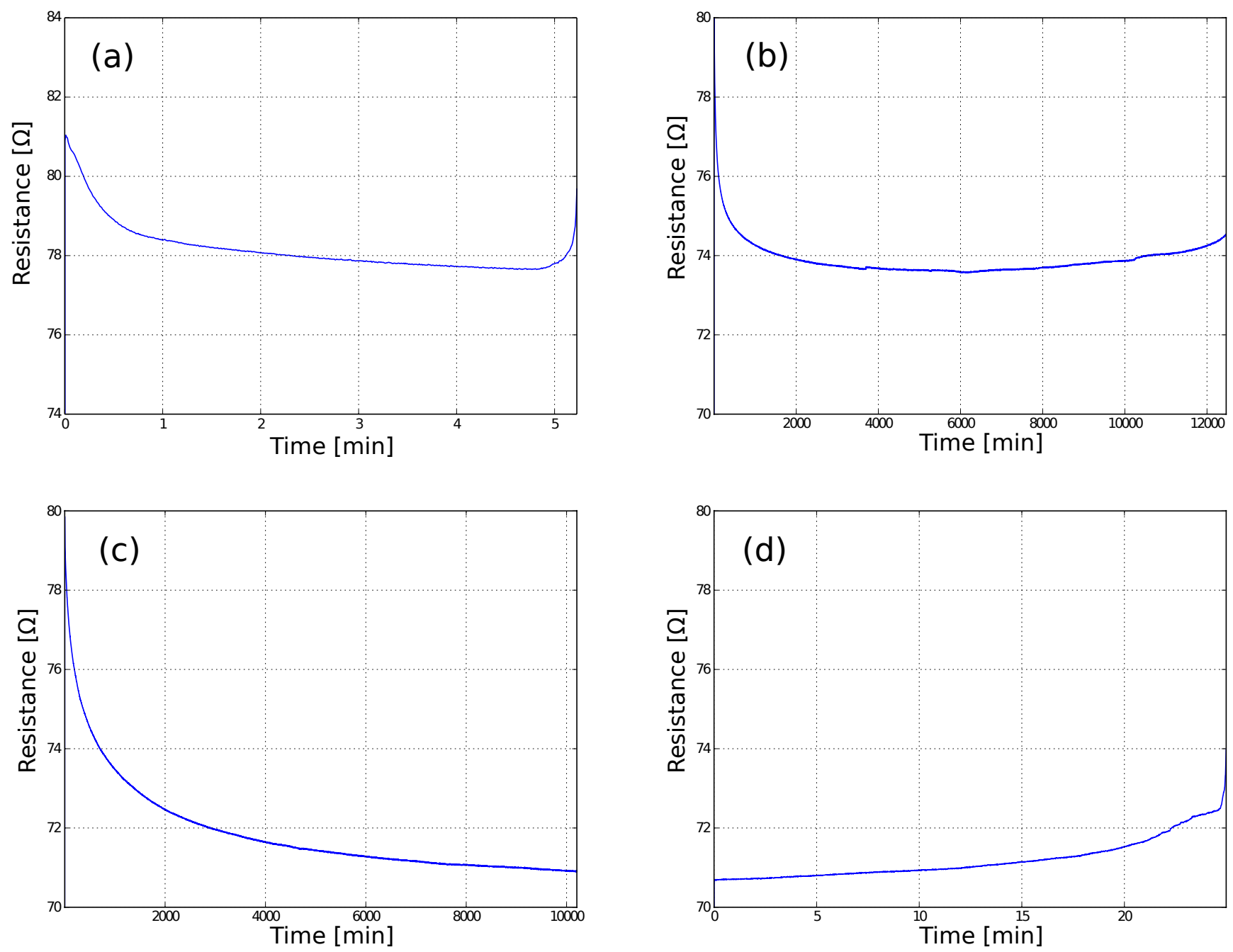

FIG. 4. $R$ - $t$ graphs of representative microheaters used in the research. (a) $P_{\mathrm{h}}=100 \mathrm{~mW}$ under DC current stress. (b) $P_{\mathrm{h}}=100$ $\mathrm{mW}$ under AC current stress. (c) and (d) AC and DC measurements, respectively, of the same microheater at $P_{\mathrm{h}}=90 \mathrm{~mW}$. The AC measurement was terminated and continued under DC bias.

TABLE I. Results of time-to-failure measurements of microheaters under AC current stress. Numbers are assigned to measurements for text references.

\begin{tabular}{cccc}
\hline \hline no. & $P_{\mathrm{h}}[\mathrm{mW}]$ & $\tau_{A C}[\mathrm{hrs}]$ & $\tau_{A C} / \tau_{D C}$ \\
\hline$\sqrt{a}$ & 90 & 170 & 153 \\
$2 \sqrt{a}$ & 90 & 137 & 123 \\
3 & 100 & 208 & 2800 \\
4 & 100 & 122 & 1600 \\
5 & 100 & 129 & 1700 \\
\hline \hline
\end{tabular}

a These measurements were aborted prior to heater destruction.

Measuring the time-to-failure of the heaters under AC current stress turned out to be very time consuming as their lifetime was extended significantly. Two heaters were measured under AC current stress at $P_{\mathrm{h}}=90 \mathrm{~mW}$ and three were measured at $P_{\mathrm{h}}=100 \mathrm{~mW}$. The results are listed in table I. It is apparent from the values in the last column of table I, which holds comparison of the time-to-failure of microheaters of AC and DC current stress, that the time-to-failure of the heaters increased at least by a factor of $10^{3}$. The heaters measured under $90 \mathrm{~mW}$ power were stopped manually since it would have taken about 7 weeks of measuring each heater to wait for a thousandfold increase of the time-to-failure, assuming a similar lifespan extention as for the $100 \mathrm{~mW}$ case. The results of our measurements display a dramatic increase in the time-to-failure of the microheaters under $\mathrm{AC}$ current stress compared to DC current stress, in agreement with ref. 17.

Figure 4 (c) displays results of a $90 \mathrm{~mW}$ measurement under AC stress. It displays the same initial behavior as described above, but it was terminated after about 7 days, the resistance slope still gradually decreasing. Based on the shape of the resistance curves and the location of the resistance minima with respect to breaking time one could predict that under these conditions this particular microheater would have lasted at least another week. However, we decided to expose this same microheater to DC bias to see how it fared. This is shown in fig. 4 (d). It lasted almost 25 minutes, i.e. about a third of the mean time-to-failure of as-grown samples at 
$90 \mathrm{~mW}$ and DC bias. Note how the simple act of turning off the $\mathrm{AC}$ bias and turning on again a DC bias abruptly changes the slope of resistance versus time from negative to positive. Also note that the initial resistance in (c) is around $80 \Omega$, while in $(d)$ it starts at $71 \Omega$, i.e. where it left off in (c). This shows that the changes in resistance are permanent, i.e. an irreversible change takes place in the wires. This contradicts previous results, e.g. in ref. 20 on $\mathrm{Cu}$ interconnects. We belive this is caused by graingrowth enabled by self-heating annealing in our wires. This effect is studied further in ref. 21,

\section{CONCLUSIONS}

We have built a biasing circuit that is computer controlled to regulate power dissipation in microwires, i.e. microheaters. This is important in order to maintain a constant temperature of the heaters, to stabilize emission intensity and radiation spectrum. We have used our setup to investigate the electromigration affecting our microheaters in terms of DC and AC time-to-failure measurements. Heaters were regulated both using power $P_{\mathrm{h}}$ $=90 \mathrm{~mW}$ and $P_{\mathrm{h}}=100 \mathrm{~mW}$, corresponding to about $440^{\circ} \mathrm{C}$ and $490^{\circ} \mathrm{C}$, respectively and were measured both under DC and AC current stress. While the measurements at $P_{\mathrm{h}}=90 \mathrm{~mW}$ proved unfeasible due to long lifetime in the AC case, the measurements at $P_{\mathrm{h}}=100$ $\mathrm{mW}$ showed that the AC time-to-failure of microheaters increased at least by a factor of $10^{3}$ compared to the corresponding DC time-to-failure.

\section{ACKNOWLEDGMENTS}

This work was partially supported by The Icelandic Research Fund grant no. 120002023. We would like to thank Pauline Renoux for help with obtaining the SEM images.

${ }^{1}$ S. Ingvarsson, L. J. Klein, Y.-Y. Au, J. A. Lacey, and H. F. Hamann, Opt Express 15, 11249 (2007).

${ }^{2}$ Y.-Y. Au, H. Skulason, S. Ingvarsson, L. Klein, and H. Hamann, Phys. Rev. B 78 (2008).

${ }^{3}$ P. Renoux, S. Æ. Jónsson, L. J. Klein, H. F. Hamann, and S. Ingvarsson, Opt Express 19, 8721 (2011).

${ }^{4}$ G. Vasile, M. Arikan, and S. Ingvarsson, J. Phys. D: Appl. Phys. 45, 445106 (2012).

${ }^{5}$ M. Born and E. Wolf, Principles of Optics, seventh edition ed. (Cambridge University Press, 2005).

${ }^{6} \mathrm{~S}$. Æ. Jónsson, Nonlinear thermal electric analysis of platinum microheaters, Master's thesis, University of Iceland (2009).

${ }^{7} \mathrm{~J}$. Lienig, in SLIP '05 (ACM Press, New York, New York, USA, 2005) pp. 39-46.

${ }^{8}$ J. Black, IEEE Transactions on Electron Devices 16, 338 (1969).

${ }^{9}$ D. Gardner, J. Meindl, and K. Saraswat, IEEE Transactions on Electron Devices 34, 633 (1987).

${ }^{10} \mathrm{~J}$. Clement, IEEE Transactions on Device and Materials Reliability 1, 33 (2001).

${ }^{11} \mathrm{~K}$. Banerjee and A. Mehrotra, IEEE Circuits and Devices Magazine 17, 16 (2001).

12 J. Lloyd, Semiconductor science and technology 12, 1177 (1997).

${ }^{13}$ J. Lloyd, Journal of Physics D: Applied Physics 32, R109 (1999).

${ }^{14}$ R. L. de Orio, H. Ceric, and S. Selberherr, Microelectronics Reliability 50, 775 (2010).

${ }^{15}$ A. S. Oates, Proc. 31st Int. Rel. Phys. Symp. , 297 (1993).

${ }^{16}$ B. Liew, N. Cheung, and C. Hu, in Reliability Physics Symposium, 1989. 27th Annual Proceedings., International (1989) pp. $215-219$.

${ }^{17} \mathrm{~J}$. Tao, N. Cheung, and C. Hu, Electron Device Letters, IEEE 14, 554 (1993).

${ }^{18}$ J. Tao, K. Young, N. Cheung, and C. Hu, IEEE Transactions on Electron Devices 40, 1398 (1993).

${ }^{19}$ C. Kittel, Introduction to Solid State Physics, 7th ed. (John Wiley and sons, 1995).

${ }^{20}$ M. H. Lin and A. S. Oates, Proc. Interconnect Technology Conference (IITC) , 1 (2013).

${ }^{21}$ O. Elíasson, G. Vasile, and S. Ingvarsson, Journal of Physics D: Applied Physics 51, 265303 (2018). 